\title{
A MODULAR DESIGN OF A WALL-CLIMBING ROBOT AND ITS MECHATRONICS CONTROLLER
}

\author{
N.S. Tlale ${ }^{1}$ \\ Department of Industrial and Systems Engineering \\ University of Pretoria, South Africa \\ ntlale@csir.co.za
}

\begin{abstract}
The modular design of a wall-climbing robot, implementing two articulated legs per module (biped robotic modules), is presented in this paper. Modular design improves a wall-climbing robot's manoeuvrability and flexibility during surface changes or while walking on uneven surfaces. The design of the articulated legs uses four motors to control the posture of the vacuum cups, achieving the best possible contact with the surface. Each leg can contain more than five sensors for effective feedback control, and additional sensors such as gyros, CCD sensors, etc, can be fitted on a module, depending on the robot's application. As the number of modules used in the design of the robot is increased, the number of actuators and sensors increases exponentially. A distributed mechatronics controller of such systems is presented.
\end{abstract}

\section{OPSOMMING}

Modulêre ontwerp van 'n muurklim-robot met twee geskarnierde bene per module (twee-benige robotmodules) word in hierdie artikel weergegee. Modulêre ontwerp verbeter die muurklim-robot se beweeglikheid en aanpasbaarheid tydens veranderings in die loopvlak of terwyl dit loop op ongelyke oppervlaktes. Ontwerp van geskarnierde bene implementeer vier motors wat die oriëntasie van vakuumsuigdoppe beheer om die bes moontlike kontak met die loopvlak te handhaaf. Elke been kan meer as vyf sensors hê vir doeltreffende terugvoerbeheer, en bykomende sensors soos giroskope, CCD sensors, ens. kan by 'n module gevoeg word soos die toepassing van die robot dit mag vereis. Soos die aantal modules wat in die ontwerp van die robot gebruik word, toeneem, neem die aantal aktiveerders en sensors eksponensiëel toe. 'n Verdeelde megatroniese beheerder van sulke stelsels word aangebied.

\footnotetext{
${ }^{1}$ The author was a staff member at the Department of Industrial and Systems Engineering at the time of writing this paper.
} 


\section{INTRODUCTION}

Repetitive tasks have been automated using robotic systems in order to reduce operational time and to improve quality and flexibility of manufacturing systems. Most such tasks and their environments require horizontal surfaces. However, there are many tasks - such as cleaning high-rise buildings, inspecting and maintaining storage tanks, etc - that are normally carried out on steep slopes or vertical surfaces. These environments can be dangerous for human operators. This will inevitably reduce the efficiency and increase the cost of performing such operations. Mobile wall-climbing robots that can carry tools and equipment to perform the required tasks provide a more cost-effective solution to the problem [1]. Such robots are termed service robots by the International Service Robot Association (ISRA) [2]. They are defined as machines that sense, think, and act to benefit (or extend) human capabilities and to increase human productivity.

A mobile robot that can climb walls or other inclined surfaces and carry out various tasks must be light enough so that its weight does not strain the structure, yet rugged enough to work in an exterior environment, and powerful enough to carry the necessary payload. It must also have the ability to climb over obstacles (since surfaces like the walls of buildings will normally have protrusions such as pipelines, window frames, etc) and to manoeuvre reliably within an undefined environment. It must be able to change surfaces - for example, changing between perpendicularly juxtaposed walls - or to manoeuvre on uneven surfaces, such as curved walls. Clearly, wall-climbing robots do not need to be able to undertake all of these tasks: some applications may require only one or two such capabilities. In this paper, the modular design of a wall-climbing robot is presented in order to solve these problems.

The developed modular wall-climbing robot implements biped robotics systems as its modules. Each biped robotic system consists of two articulated legs. The minimum number of actuators and sensors in one biped module is sixteen. Adding modules exponentially increases the number of actuators to be controlled and the number of sensors to be monitored. This presents challenges when designing the controller for such mechatronics systems. Implementing a central microcontroller system (embedded control system) will result in hard-to-trace, untidy connection wires. Troubleshooting or installing software code to control the robot will be very difficult to develop. Real-time control of the developed wall-climbing robot becomes complicated when too many actuators and sensors are used. In this paper, a distributed mechatronics controller for a system is presented. The controller is based on Controller Area Network (CAN) technology. The disadvantage of distributed control systems is that delays are introduced into the systems because of distributed control architecture. This includes problems with timing, such as the lag effect of zero-order hold $(\mathrm{ZOH})$, and with motion control. Problems of time variations can also be partially tackled in control design - for example, by using robust control so that deviations from nominal timing can be tolerated [3]. 


\section{CURRENT WALL-CLIMBING ROBOTIC SYSTEMS}

Research into robot mechanisms that enables service robots to move along vertical surfaces has been carried out since the late 1980s and early 1990s. As early as 1992, a three-legged robot (a gait) that climbed between two ladders was described in [4]. Much effort thereafter was concentrated on developing the pneumatic systems that enabled wall-climbing robots to adhere safely to vertical surfaces. Many different wall-climbing robots have been developed since then. (An exhaustive list of the developments in the area of wall-climbing robots will not be given in this paper, but can be found in [5], [6] and [7].) A review of wall-climbing robots that are currently being researched and implemented in industry is given next.

One recently designed robot is the micro-robot "The Crawler", designed in the Robotics and Automation Laboratory of Michigan University [8]. It uses two suction cups and has a light payload. Another is the "MRWALLSPECT-I", which uses six suction cups, three operating at one time [9]. It was designed in the Mechatronics Laboratory of SungKyunKwan University. It is slow and cannot be used for applications requiring high or moderate speeds. The most recent successful wallclimbing robot is "The Climber", commercially manufactured by Clarifying Technologies Ltd [10]. The Climber is mostly suited to robotic applications such as law enforcement, security, and inspection, due to its low payload of $0.45 \mathrm{~kg}$. The Climber uses a versatile six-wheeled position-traction drive train and a patented "vortex attractor" technology to pull itself on to any surface: wall board, plaster, brick, cinder block, magnetic and non-magnetic surfaces, etc. Although it can make the transition from a horizontal (ground) surface to a vertical one and back, it cannot go from a vertical to an inverted surface or directly around corners unassisted. It has high power requirements, since the motor creating the suction must always be turned on to create the suction force.

\section{MECHANICAL DESIGN OF BIPED MODULES}

The wall-climbing robot developed in this paper is inspired by insect anatomy. Insects have segments that allow their bodies to be flexible. This allows insects to manoeuvre easily around objects that have complex shapes, and to have stable motion while climbing vertical surfaces. The robot implements articulated legs that consist of four joints each, each actuated by a DC servomotor (motor 1 to motor 4). This arrangement gives each leg three degrees of freedom (DOF) (refer to Figure 1). Motor 4 is a redundant motor - that is, it does not contribute additional DOF. Its purpose is to maintain a certain pose of the vacuum suction cup when the leg is making contact with the surface.

Other walking hexapod or quadruped robots that have been developed normally use a parallelogram mechanism to achieve this. They have only one motor per articulated robot leg, and have only one body. Although one-motor-per-leg and one stiff body is enough to achieve locomotion, it is not enough to achieve smooth motion with minimised friction. This is because all the legs are mounted on one frame. As the legs of the robot are moving to cause change in the displacement of the robot's body, the robot frame is changing its posture and orientation. Since the frame is not elastic, 
it does not bend to allow the legs that have made contact with the ground to maintain their original posture when they first made contact with the ground. This causes the part of the legs which is in contact with the ground to slip and thus to lose efficient contact with the ground. Any movement of the robot's frame is propagated to the tip of the articulated leg making contact with the surface.

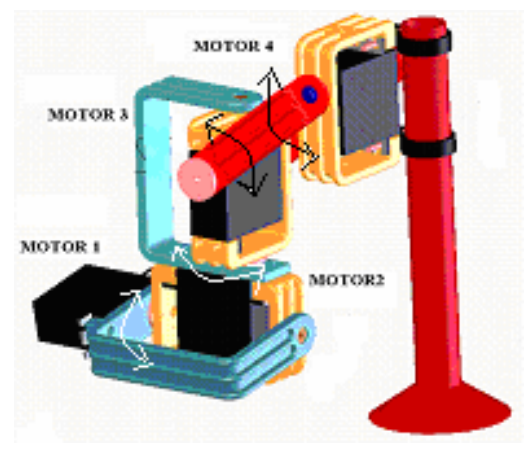

Figure 1: Articulated leg of the biped module

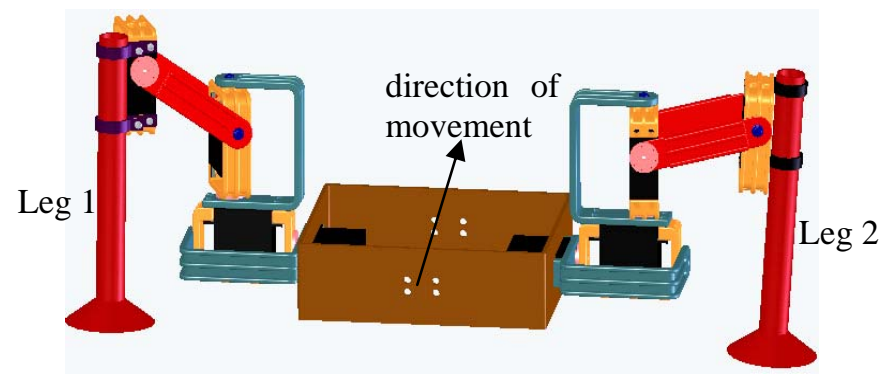

Figure 2: The biped module for wall-climbing

The first motor is attached to a base, which in turn is attached to the body frame of the biped module (Figure 2). The purpose of the motor is to rotate the complete leg, enabling the robot to change walking surfaces - for example, from floor to wall. The first motor drives the second motor's housing. The purpose of the second motor is to move the rest of the leg forwards and backwards during locomotion. The second motor drives the housing of the third motor. The purpose of the third motor is to move the fourth motor and the suction cup up and down during locomotion. The fourth motor and the suction cup are attached to the same housing. The purpose of the fourth motor is also to move the suction cup up and down during the locomotion of the robot. The third and the fourth motors act together to achieve smooth contact between the suction cup and the surface that is being moved on.

In order to assemble a wall-climbing robot that consists of any number of biped modules, each biped module (except the first module in the assembly) is fitted with a motorised revolute joint at the front. The joint gives the two modules attached to it one DOF. This gives the developed wall-climbing robot the ability to change surfaces easily (Figures 3 (a) and (c)). The overall motion of the robot can be achieved by moving one leg at a time (using only one biped module), or by a combination of two legs at a time (in a quadruped robot with two biped modules), or 
by a combination of three legs at a time (in a hexapod robot using three modules), and so on. The robot can turn around, rotating about its centre using a combination of two legs during locomotion. These different combinations of leg movement are called gaits. Unlike other developed wall-climbing robots, this capability makes the robot overcome obstacles quickly.

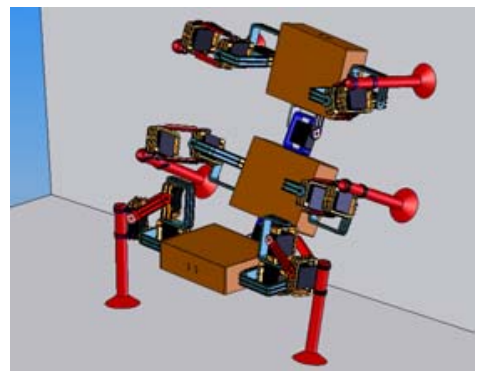

(a)

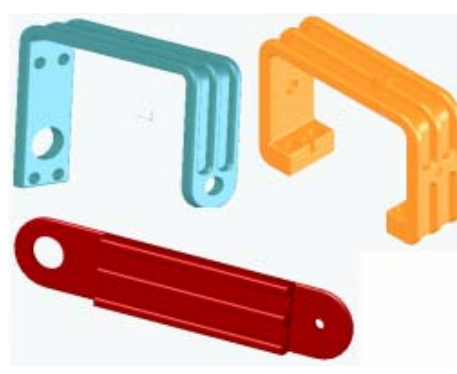

(b)

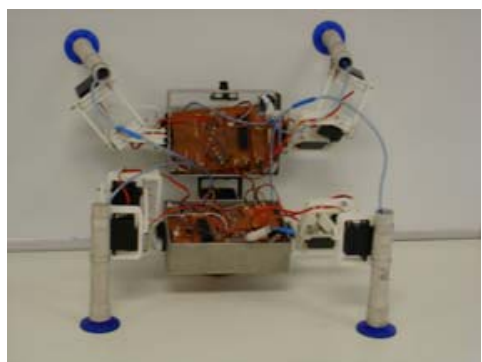

(c)

\section{Figure 3: (a) Concept of modular-wall-climbing robot. (b) Design of the brace holders and connectors. (c) The developed wall-climbing robot.}

\subsection{Improving stability}

In order to ease the problem of controlling the wall-climbing robot, inherent stability is taken into consideration in the mechanical design. The biological mechanisms of many specialised climbing creatures have shown one common characteristic: the limbs of the creature leave the body in such a way that the creature's belly may be close to the ground while it is in motion. In beetles, for example, this is achieved by a wide spread of the legs, often exceeding the body width by more than three times. In spiders the body hangs below the 'knees' and the limbs can adduct to a little more than the body width and still maintain stability [1]. An up-and-over spider-like arrangement appears to have better potential for negotiating obstacles. The body can be raised and lowered, and the reach of the legs can easily be made to exceed body length. Such an arrangement will help in negotiating obstacles and provide the mobile robot with the desired stability. In the proposed wall-climbing robot, this is achieved by raising the knee joint of the robot leg, the joint of the second and third motors, from the body level of the biped robot module, and increasing the height of the rod that connects the suction cup to the rest of the leg, to give the module strength and robustness (as shown in Figure 2). If the imagined direction of the biped module's movement is away from the reader and into the paper, then Leg 1 is the left leg and Leg 2 is the right leg.

It has been shown that a walking robotic system using $n(>2)$ articulated legs to walk on horizontal ground needs a minimum of three legs to be in contact with the ground - that is, having a tripod of support, as in a three-legged chair - in order to achieve static stability [11]. The mass-centre of a walking robot must fall inside the triangle of support formed by the three legs on the ground; otherwise it is statically unstable and will fall over. In the quasi-static gait of a robot or animal, the mass-centre moves with respect to the position of the legs on the ground, and the probability of falling increases as the centre of mass approaches the edge of the triangle of support. This 
probability has been quantified for all $n$-legged-walking robotic systems by calculating the longitudinal stability margin, the shortest distance from the masscentre to the boundary of the support pattern in front of or behind the robot. The longitudinal stability margin $(S)$ of a wave gait (i.e., a regular, symmetrical gait) normalized for stride length is described by:

$$
S=\left(n_{\text {legs }} / 4\right) \beta-(3 / 4)
$$

where $\beta$ is the duty factor, the fraction of a stride period that a leg is in the support phase; and $n_{\text {legs }}$ represents the number of legs [12]. When $S<0$, the robot is statically unstable. This criterion for static stability is applicable to robots on horizontal surfaces whose articulated legs are not adhered to the walking surface. For robots that are walking on inclined surfaces and that are using some form of adherence to the surface, the moments about the mass-centre must balance as well. The control program of the developed modular wall-climbing robot takes this into consideration in order to achieve static stability of the robot at all times. The dynamic stability of the wall-climbing robot is not considered, due to low walking speeds.

\section{VERTICAL SURFACE ATTRACTION AND TRANSLATIONAL METHODS}

One of the first steps in designing a wall-climbing robot is to decide on the technique that will be used to adhere a robot safely to vertical surfaces. Three techniques have been implemented in different designs for wall-climbing robots:

- Vacuum suction, implementing pneumatic suction cups: used on non-ferrous, smooth surfaces such as glass [13];

- Magnetic suction, using an electromagnet or a permanent magnet: used on ferrous or magnetic surfaces, such as boilers [14]; and

- Vortex attraction, implementing a mechanical device that forms a vortex: used on any surface [15].

Another design consideration for wall-climbing robots is the technique that is employed to achieve its motion. Techniques that have been used are: articulated legs, sliding frames, tracked propulsion, and wheeling mechanisms. Articulated legs move by keeping a grip on the surface while moving free legs further up the surface. By repeating this motion, multi-legged robots can climb [16]. Sliding frame robots have two or more body platforms that are linked to each other by a prismatic joint so that they can slide against each other. One platform of the body stays gripped in place while the other releases its grip from the surface, slides itself in the desired direction, and then reattaches [17]. By adding the ability for the central platform to rotate, sliding frames can move both up and down, and left and right. This allows the robot to have more degrees of motion for adapting to complex wall geometries. Tracked propelled vehicles use a loop of track to grip the ground and move forwards or backwards. Sometimes this motion can help robots change surfaces [18]. The movement of the climbing robots using walking or translating mechanisms is discontinuous due to the operating mode of sticking-moving-sticking. To overcome this shortcoming, mechanisms using wheels or crawlers have been employed for some wall-climbing robots [19], [20]. 
The design of the service robot described in this paper uses vacuum suction created by vacuum pumps and vacuum cups. The high strength to mass ratio of vacuum systems is desirable, since it improves the safety of wall-climbing robots. Articulated legs are used to improve the developed wall-climbing robot's manoeuvrability and flexibility. The robot's ability to changes surfaces is improved by the modular design (Figure 3 (a)). Each articulated-leg has a suction cup that helps the robot cling to smooth surfaces. This gives the robot the ability to walk on vertical surfaces, as enough vacuum pressure is maintained in the suction cups. Two miniature suction pumps are used to create and maintain vacuum in the suction cups when they make contact with a smooth surface. Each suction pump has been tested and can carry more than $3 \mathrm{~kg}$. The pressure in the suction cups is regulated by miniaturised microvalves. The valves can set the pressure in the vacuum cups to be either vacuum or atmospheric pressure. The use of vacuum suction cups limits the application of the developed wall-climbing robot to smooth surfaces only.

The body frame has been constructed of aluminium to minimize mass. All parts of the designed robot have been manufactured on the Dimension rapid prototyping machine. The design of the brace holders and the connectors is shown in Figure 3 (b). The ribs were added to the robot parts to give strength and robustness. One module of the developed robot prototype weighs just over $2 \mathrm{~kg}$. The body frame also houses the electronics, sensors and all the other associated equipment, depending on the application tasks.

\section{SENSOR AND ACTUATOR ARCHITECTURE}

The tip of each leg is fitted with an infra-red touch sensor, which indicates when the suction cup has made contact with the surface. Limit switches are used on each leg to stop Motor 3 from over-rotating and colliding with Motor 2. Each leg is also fitted with a pressure sensor that monitors the pressure in the pneumatic system of each leg. The pressure in the suction cups is controlled by micro-switches.

The front of the first biped module is fitted with one ultrasonic sensor and one CCD sensor, and a gyroscope sensor is fitted on its centre. Ultrasonic sensors are used for object detection. CCD sensors are used for data visual collection, and help with home position navigation. A gyroscope (or inertial sensor) is used for improving stability and for navigation. One independent biped module consists of nine motors, two micro-valves, two pressure sensors, two infra-red touch sensors, two limit switches, one ultrasonic sensor, one CCD camera, and one gyroscope sensor - that is, eleven outputs to be controlled and nine inputs to be monitored. The high number of sensors and actuators used requires that a distributed controller be used (for reasons given earlier in this paper). An MCP2515 CAN controller from Microchip [20] is used as a stand-alone distributed control network. PIC18F442 microcontrollers are used as local controllers on the network's nodes.

\section{DISTRIBUTED MECHATRONICS CONTROLLER IMPLEMENTING CAN}

The robot control architecture is divided into time-critical and event-based control strategies. Control functions such as navigation and motion planning are time- 
critical, while control functions such as path planning and object avoidance are event-based. To achieve reliable and adequate control of the robot, the Controller Area Network (CAN) is implemented as a mechatronics distributed controller of the developed modular wall-climbing robot. CAN has been widely used within the European automotive industry, and the decision to use CAN from the US and Japan automotive industry is guaranteed because of the availability of CAN solutions more than fifty controllers from low-cost devices to high-end chips from more than fifteen manufacturers. Figure 4 shows the architecture of a mechatronics distributed controller implementing MCP2515 CAN for controlling a modular wall-climbing robot that consists of $n$ modules. Figure 5 shows the CAN hardware that was used on each biped module.

Controller Area Network (CAN) is a serial bus system especially suited to interconnect smart devices to build smart systems or sub-systems. The CAN bus has been successfully implemented to control PloyPod, which is a modular selfreconfigurable robot [21]. Due to the high number of robot modules used, special higher level software layers were designed. In our application, for the developed modular robot to be practical, fewer than six modules are sufficient - that is. scaling is not a problem.

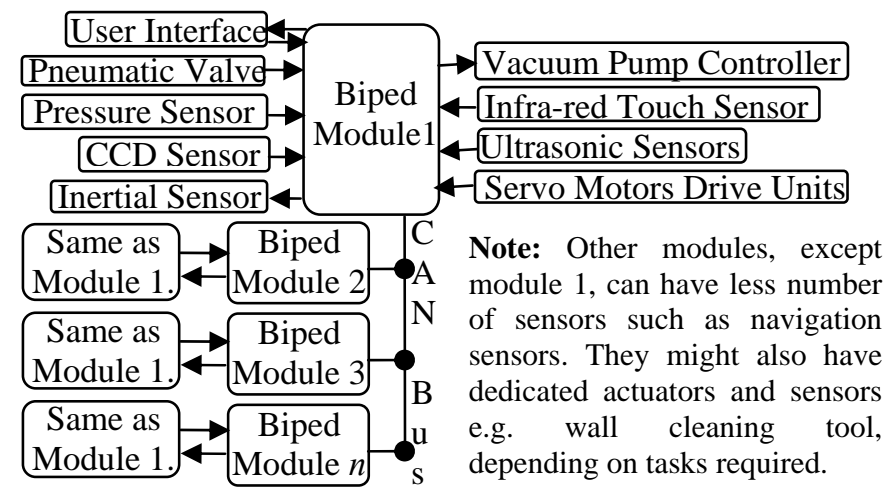

Figure 4: The hardware structure of the control system using stand-alone CAN

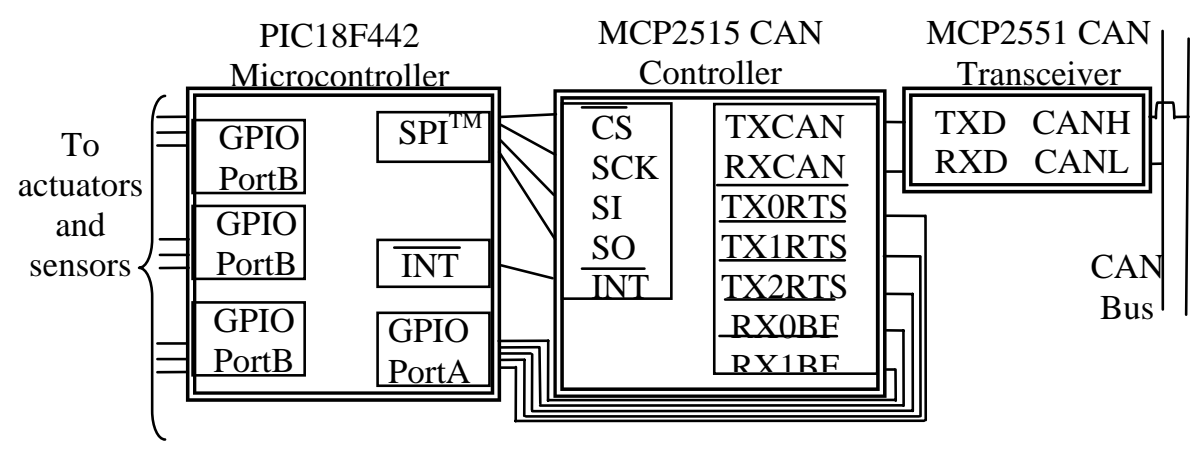

Figure 5: CAN hardware implemented on each module

No high level software is needed to achieve proper implementation of the CAN bus. The attributes of a Controller Area Network (CAN) are: 
- the multi-master capabilities that allow smart and redundant systems to be built without the need for a valuable master;

- the broadcast messaging that is the first element that guarantees $100 \%$ data integrity, as any device within the network uses the very same information;

- the sophisticated error detecting mechanism and the retransmission of faulty messages - the second element that guarantees $100 \%$ data integrity;

- only layers 2 (Data Link Layer) and 1 (Physical Layer) of the International Standardisation Organisation/Open Systems Interconnect (ISO/OSI) hierarchical layered structure [22], [23]; and

- a CAN message data frame that uses a standard data frame, and consists of eleven message identifier bits and eight data bytes.

The local microprocessor controls the local actuators and monitors the local sensors. It directly controls the individual movements of each of two articulated legs of the biped modules. It can indirectly control remote actuators and monitor remote sensors by sending and receiving correctly addressed messages on the CAN bus. Only messages with message identifiers that have been programmed to be accepted by the local MCP2515 can be used as control data for local actuators. Similarly, the local microprocessor can control remote actuators (on a different CAN node) if the remote MCP2515 is programmed to accept the messages with its message identifiers.

Synchronisation of the movement of the legs is achieved by sending synchronizing messages. These are divided into three hierarchical layers: top, middle and bottom. Figure 6 illustrates the different layers, their priority, and their time requirements in respect to control. Priority of CAN messages and fast control times increase with the lower hierarchical layer. In CAN, message priority increases as the values of message identifiers become low.

The top layer contains messages that represent the current task of the robot - e.g., robot searching for a certain target using a CCD sensor. This task may be initiated by the user-interface unit attached to the first module, or by any other node when the robot is operating autonomously (user-interface consists of a wireless radio control unit). This message is then broadcast on the CAN bus to all the CAN nodes (or robot's modules). Message identifiers are used to identify the message's nature and origin. CAN has an internal error handling mechanism that handles any message errors during transmission. A CAN message that experiences error during transmission is re-sent. Thus, it can be known whether the CAN has received the task message or not, and the next step in the control procedure can be implemented. CAN nodes that do not receive a transmitted message will not acknowledge receipt of that message. This indicates to the node that initiated a message that the message it tried to transmit on the CAN bus experienced an error. The top layer is event-driven and is not affected by the time delays. Any microprocessor in the distributed control system can be used to achieve stable control of the top layer.

The middle layer contains a sequence of movements that are required to achieve the task. After each CAN node has received the message that represents the task to be done, it will then broadcast a message that contains the sequence of events that are to be done to achieve the required task - that is, task planning messages are generated. 
The CAN node that initiated the message can indirectly be determined by the message identifier. The message contains the sequence of leg movements to be done. It also contains the information about when and how the specific task actuator is to be controlled if the robot is fitted with that actuator - e.g. a window cleaning brush actuator. If there are many CAN nodes, this step can be omitted since it will increase the time delays in achieving the robot's tasks. Since all the CAN nodes have the same competency, it can be assumed that all the nodes will produce the same task planning schedules. Using the example given previously, one of the strategies for searching for a target using a CCD camera is to rotate the robot once in a clockwise direction until the target is in the view of the CCD sensor. The CAN message structure is as follows: (message identifier, (first module to implement control(what is to be controlled, what is to be done)), (second module to implement control(what is to be controlled, what is to be done)), ...). If all the contents of the messages are the same, then the robot is controlled according to the messages received and sent.

\begin{tabular}{|c|c|c|}
$\begin{array}{c}\text { Distributed control, any } \\
\text { microprocessor on the } \\
\text { CAN network can be used } \\
\text { to achieve control }\end{array}$ & $\begin{array}{c}\text { Top Layer } \\
\text { Task messages - e.g. } \\
\text { reference target, object } \\
\text { avoidance, target steering, } \\
\text { home position, etc. }\end{array}$ & $\begin{array}{c}\text { Low priority CAN } \\
\text { message, even-based } \\
\text { control, can accommodate } \\
\text { large time delays }\end{array}$ \\
\hline & $\begin{array}{c}\text { Middle Layer } \\
\text { Task planning - e.g. move } \\
\text { forward, move backward, } \\
\text { rotate clockwise, etc. }\end{array}$ & \\
\hline $\begin{array}{c}\text { Only local CAN node } \\
\text { microprocessor used to } \\
\text { achieve control. }\end{array}$ & $\begin{array}{c}\text { Task implementation - } \\
\text { B.g. which legs must be } \\
\text { moved in what sequence? } \\
\text { Object-detection, task } \\
\text { error detection, etc. }\end{array}$ & $\begin{array}{c}\text { High Priority CAN } \\
\text { message, time-critical } \\
\text { tasks, real-time control, } \\
\text { cannot accommodate large } \\
\text { time delays }\end{array}$ \\
\hline
\end{tabular}

Figure 6: Hierarchical layer for CAN

The bottom layer controls and monitors the state of the actuators and sensors of the robot to achieve synchronised control. The lower layer is time critical, and is controlled by a local microprocessor. In a modular robot that contains two biped modules, clockwise rotation of the wall-climbing robot is achieved by moving the left legs to the front, one articulated leg at a time (say, Leg 1 and Leg 2), and moving the right legs to the back, one articulated leg at a time (say Leg 3 and Leg 4) (Figure 2). One sequence of leg movements to achieve this is: (module1 (leg 1 (forward)), module 2 (leg 1(forward)), module 1(leg 2 (backward), module 2(leg 2 (backward))). When module 1 is finished moving Leg 1 forward, it then sends a message on the CAN bus that Leg 1 has finished forward motion. Then module 2 starts moving its Leg 1 forward. When this has been finished, module 2 sends a CAN message that Leg 2 is finished moving forward, and so on until the task has been finished. If an error is 
encountered while the leg is being moved - for example, pressure loss in the suction cups due to an irregular surface - an error message regarding this task is sent on the CAN bus before the task is aborted and a new control sequence has been generated.

\section{DISCUSSION AND CONCLUSION}

A distributed mechatronics controller is used to control the biped modules of a wall climbing robot. A distributed controller simplifies the controller design, especially if the system to be controlled has many actuators and sensors. It allows the intelligence to be embedded close to the actuator or sensor, thus improving the response time and performance of the controller - that is, giving near real-time control.

The developed wall climbing robot consists of many actuators and sensors. A CAN network is an ideal solution for developing a distributed, real time mechatronics controller. It is an alternative to $\mathrm{I}^{2} \mathrm{C}$ bus, and more advanced than $\mathrm{I}^{2} \mathrm{C}$ bus. CAN facilitates adequate control of the event-based and time-critical tasks of the developed wall-climbing robot. CAN messages that are used in this project are divided into three hierarchical messages, depending on the type (or urgency) of the message. The hierarchy also represents the priority of the message on the CAN bus.

The developed robot also used biped robotic modules. Assembly of a number of biped robotic modules to achieve a modular wall-climbing robot is achieved by one DOF revolute joint between two consecutive modules. Since the connection between the consecutive modules is flexible, the developed wall-climbing robot can safely change surfaces that are at an angle to each other - for example, moving from the floor to a wall.

\section{REFERENCES}

[1] B. L. Luk, A. A. Collie and J. Billingsley, 1991, "Robug II: an intelligent wall-climbing robot", Proc. IEEE Int. Conf. on Robotics and Automation, Vol. 3, pp 2342-2347.

[2] Pransky, J., 1996, "Service robots - how we should define them?", Service Robot: An International Journal Volume 2, Number 1, pp. 4-5.

[3] Chen, D-. J., 2001, “Architecture for Systematic Development of Mechatronics Software Systems”, Licentiate Thesis, ISSN 1400-1179, Mechatronics Lab, Department of Machine Design, Royal Institute of Technology, KTH, Stockholm, Sweden.

[4] Madhani, A. and Dubowsky, S., 1992, "Design and Motion Planning of Multi-Limb Robotic Systems: The Force Workspace Approach”, Proceedings of the 1992 ASME Design Technical Mechanisms Conference, Phoenix, AZ.

[5] Ultimate Real Robots Magazine, 2003, "Robots in Action: Climbing Robots", Issue 36, Eaglemoss Publications, UK, or www.realrobots.com.

[6] Ultimate Real Robots Magazine, 2003, "Robots in Action: Climbing Robots", Issue 37, Eaglemoss Publications, UK, or www.realrobots.com.

[7] "Special Issue: Climbing and walking robots", Industrial Robot: An International Journal, Volume 31 Number 2, 2004. 
[8] Xiao, J., Minor, M., Dulimarta, H., Xi, N., Mukherjee, R. and Tummala, R. L., 2001, "Modelling and Control of an Under-actuated Miniature Crawler Robot", Proceedings of the 2001 IEEE/RSJ International Conference on Intelligent Robots and Systems, Maui, Hawaii, USA.

[9] Kang, T., Hyungsuk, K., Taeyoung, S. and Choi, H., 2003, "Design of Quadruped Walking and Climbing Robot”, IEEE/RSJ International Conference on Intelligent Robots and Systems.

[10] Illingworth, L. and Reinfeld, D., 2001, "The Vortex Attractor: US Patents \#6565321 and \#6497553”, Vortex Holding Company, United States Patent and Trademark Office, USA.

[11] Ting, L. H., Blickhan, R., and Full, R. J., 1994, "Dynamic and Static Stability in Hexapedal Runners.”, J. exp. Biol. 197, Great Britain, London, pp. 251-269.

[12] Song, S. M, 1984, "Kinematic optimal design of a six-legged walking machine.”, PhD Dissertation, Ohio State University, Columbus, Ohio.

[13] Briones, L., Bustamante P. and Serna, M.A., 1994, "Wall-climbing robot for inspection in nuclear power plants.”, Proc. IEEE Int. Conf. on Robotics and Automation, pp. 409-1414.

[14] Naitou, S., 1992, "Wall surface robot with magnetic crawlers", Journal of Japanese Society of Robotics, Vol.10, No.5.

[15] http://www.clarifyingtech.com/public/robots/robots_public.html, retrieved 06/05/2004.

[16] Nagakubo, A. and Hirose, S., 1994, "Walking and running of the quadruped wall-climbing robot”, Proc. IEEE Int. Conf. on Robotics and Automation, pp. 1005-1012.

[17] Gradetsky, V. G., Rachkov, M. Y. and Nandi, G. C., 1992, "Vacuum pendipulators for climbing robots”, Proc. of $23^{\text {rd }}$ ISIR, 517-523.

[18] Nishi, A. and Miyagi, H., 1994, "Wall-climbing robot using propulsive force of a propeller: mechanism and control system in a strong wind", JSME International Journal of Dynamics, Control, Robotics, Design and Manufacturing, Vol. 37, No. 3, pp. 172-178.

[19] Nishi, A., 1992, "Wheel or crawler type wall-climbing robot", Journal of Japanese Society of Robotics, Vol.10 No.5.

[20] http://www.microchip.com/, retrieved 18/07/2004.

[21] Zhang, Y., Roufas, K. and Yim, M., 2001, "Software Architecture for Modular Self-Reconfigurable Robots”, IEEE/RSJ Intl. Conf. on Intelligent Robots and Systems (IROS), Hawaii, USA.

[22] International Standard ISO 11519 -2, 1993, "Road vehicles - Low Speed Serial Data Communication - Part 2: Low-speed Controller Area Network (CAN)”, ISO Reference Number ISO 11519-2:19994 (E), First Edition, pp. $11-15$.

[23] International Standard ISO 11898, 1993, "Road Vehicles - Interchange of Digital Information - Controller Area Network (CAN) for High Speed Communication”, ISO Reference Number ISO 11898: 1993(E), First Edition $1993-11-15$. 